\title{
基于无机填料复合薄膜的摩擦纳米发电机研究进展
}

\author{
郭隐告牪 ${ }^{1}$, 陈子䀬 ${ }^{1}$, 王宏志 ${ }^{2}$, 张青红 ${ }^{3}$
}

(1. 上海工程技术大学 材料工程学院, 上海 201620; 2. 东华大学 材料科学与工程学院, 纤维材料改性国家重点 实验室，上海 201620; 3. 东华大学 教育部先进玻璃制造技术工程中心，上海 201620)

摘 要: 摩擦纳米发电机(Triboelectric Nanogenerator, TENG)是一种将微小机械能转化为电能并加以收集利用的绿 色能源器件, 具有活性材料种类广泛、器件结构简单以及易于集成等特点。较低的输出功率密度是目前阻碍其实际 应用的主要因素之一。如何通过材料组分设计与制备提高其输出功率密度及能量转化效率, 是目前该领域研究者关 注的热点问题。在摩擦纳米发电机常用的活性材料-高分子聚合物中引入功能性填料是一种简便且高效的改性方法, 不仅能够对薄膜摩擦电性能进行优化、提高输出性能, 还能够赋予其新功能, 可谓一举多得。因此, 此类复合薄膜 已广泛应用于 $\mathrm{TENG}$ 领域, 例如 $\mathrm{TiO}_{2} 、 \mathrm{SiO}_{2} 、 \mathrm{BaTiO}_{3} 、 \mathrm{ZnSnO}_{3} 、 \mathrm{MoS}_{2}$ 、石墨烯、二维黑磷等无机填料对复合材料 的性能均有不同程度的优化, TENG 的输出功率密度最高提升了数十倍。本文结合国内外研究现状, 按照填料对基 体材料表面性能以及电学性能优化作用两个方面进行阐述, 综述了复合材料薄膜在摩擦纳米发电机中的研究进展, 并展望了未来复合材料用于提高 TENG 输出性能研究的发展方向。

关 键 词: 无机填料; 复合薄膜; 表面性能; 电学性能; 输出功率密度; 摩擦纳米发电机; 综述 中图分类号: TB332 文献标志码: A

\section{Progress of Inorganic Filler Based Composite Films for Triboelectric Nanogenerators}

\author{
GUO Yinben ${ }^{1}$, CHEN Zixi ${ }^{1}$, WANG Hongzhi ${ }^{2}$, ZHANG Qinghong ${ }^{3}$
}

(1. College of Materials Engineering, Shanghai University of Engineering Science, Shanghai 201620, China; 2. State Key Laboratory for Modification of Chemical Fibers and Polymer Materials, College of Materials Science and Engineering, Donghua University, Shanghai 201620, China; 3. Engineering Research Center of Advanced Glasses Manufacturing Technology, Ministry of Education, Donghua University, Shanghai 201620, China)

\begin{abstract}
The triboelectric nanogenerator (TENG) is a kind of green power source which can harvest and transform small mechanical energy into electricity. Triboelectric nanogenerators have various active materials, simple structures, and easy to integrate with other devices. However, its relatively low output power density hinders the further practical application of TENGs. How to improve the output performance of TENGs through the modification of the active triboelectric materials is one of the hottest spots. It is a facile and effective way to introduce functional fillers into polymer substrates to fabricate composite materials, which improve the triboelectricity of pristine material and bring new functions for the device. Thus, composite films are widely used in TENGs. For example, inorganic fillers like $\mathrm{TiO}_{2}, \mathrm{SiO}_{2}, \mathrm{BaTiO}_{3}, \mathrm{ZnSnO}_{3}, \mathrm{MoS}_{2}, \mathrm{r}-\mathrm{GO}$ sheets, and nanofibril-phosphorene have been introduced into polymers to improve the output power density of TENGs by dozens of times. Based on domestic
\end{abstract}

收稿日期：2020-12-29；收到修改稿日期：2021-02-22; 网络出版日期：2021-03-12

基金项目：国家自然科学基金(51903151); 上海市“晨光计划”(19CG66) National Natural Science Foundation of China (51903151); Shanghai "ChenGuang” Project (19CG66 )

作者简介: 郭隐辚(1990-), 女, 讲师. E-mail: guoyb@sues.edu.cn GUO Yinben(1990-), female, lecturer. E-mail: guoyb@sues.edu.cn

通信作者: 张青红, 教授 E-mail: zhangqh@dhu.edu.cn ZHANG Qinghong, professor. E-mail: zhangqh@dhu.edu.cn 
and international research, this review introduces the applications of the composite film in TENGs. The improvements of TENGs induced by the fillers are discussed from two aspects: the surface property and electrical property. Finally, future challenges in developing composites based TENGs are prospected.

Key words: inorganic filler; composite film; surface property; electrical property; output power density; triboelectric nanogenerator; review

摩擦纳米发电机 (Triboelectric Nanogenerator, TENG)自 2012 年被首次报道以来, 迅速受到科研人 员的广泛关注 ${ }^{[1]}$ 。TENG 是基于摩擦起电和静电感 应的耦合作用, 将诸如人体运动 ${ }^{[2]}$ 、风能 ${ }^{[3-4]}$ 和水波 能 ${ }^{[5-6]}$ 等日常生活中的低频机械能转化成电能并收 集起来加以利用的器件。与其他能量收集技术相比, TENG 具有可使用材料广泛、制造简单、成本低廉、 安全性高等优势。目前, TENG 已被证明在柔性自供 能传感器、可穿戴设备和智能机器人等应用领域具 有极大的潜力 ${ }^{[7-10]}$ 。但是, TENG 存在输出功率密度 较低的缺点, 这是阻碍 TENG 广泛应用的主要因素 之一。影响 TENG 输出性能的因素有很多, 例如摩 擦层材料的性能、TENG 的器件结构以及测量的环 境条件等。其中, 摩擦层材料的表面性能以及电学 性能是决定摩擦电器件表面电荷密度大小的关键因 素。目前, 研究人员常用聚对苯二甲酸乙二醇酯 (PET)、聚二甲基硅氧烷(PDMS)、聚偏氟乙烯(PVDF) 以及聚四氟乙烯(PTFE)等高分子聚合物材料作为 TENG 的介电摩擦层。虽然这些材料本身具有良好 的摩擦电性能 ${ }^{[11]}$, 但是为了追求更高的输出功率密 度, 研究人员探索了各种方法, 包括摩擦层材料表面 功能化 ${ }^{[12-13]}$ 、表面图案化 ${ }^{[14-15]}$ 以及微纳米结构构筑 ${ }^{[16]}$ 等以扩大 TENG 的实际应用范围。

尽管以上这些方法有效地提高了摩擦层材料在 工作过程中的表面电荷密度, 优化了 TENG 器件的 输出性能, 但是受限于材料本身的性质, 器件的输 出性能难以进一步提升, 这极大地限制了 TENG 的 应用范围。为了解决这一问题, 设计制备复合材料 作为 TENG 介电摩擦层的研究开始不断涌现 ${ }^{[17-19]}$ 。 介电摩擦层复合材料体系的构建不仅可以改变材料 成膜后的表面性能, 还能够优化材料的电学性能, 这是一种提高 TENG 输出性能的有效策略。因此, 研究者们在摩擦层材料体系中引入各种无机填料, 构建有机一无机复合材料体系。这些活性填料主要有: 高介电性能的氧化物填料如 $\mathrm{TiO}_{2} 、 \mathrm{SiO}_{2} 、 \mathrm{BaTiO}_{3}$ 及 $\mathrm{ZnSnO}_{3}$ 等; 高捕获电子能力的碳材料如纳米碳球、 氧化石墨及石墨烯纳米片等; 功能型二维材料如 $\mathrm{MoS}_{2} 、 \mathrm{MXene} 及 二$ 维黑磷等; 新型有机-无机杂化 材料如 $\mathrm{CH}_{3} \mathrm{NH}_{3} \mathrm{PbI}_{3}$ 、金属有机框架材料等。引入这
些无机填料, 不仅能够在不同程度上提高 TENG 的 输出性能, 而且能够为器件增添多样的功能性, 成 功将 TENG 的应用领域拓展到光探测、光电催化、 物联网等众多领域。因此, 本文将从优化摩擦层 材料的表面性能及电学性能两方面来综述用于 TENG 介电摩擦层的复合材料体系的构建与应用 (图 1)。

\section{1 优化材料表面性能}

摩擦层材料在工作过程中能够产生的表面电荷 量是决定 TENG 输出性能的主要因素之一。而表面 电荷量的大小则取决于材料本身的摩擦电负性以及 摩擦层材料的有效接触表面积。其中, 虽然材料的 摩擦电负性产生原理尚存在争议, 但是已通过实验 证实其与材料表面所带功能性基团密切相关 ${ }^{[28-30]}$ 。而 对于相同的材料, 有效接触面积越大, 摩擦产生的 电荷量也越大。因此, 可以通过对材料表面形貌及 结构的设计, 达到提高表面电荷密度以及增大摩擦 接触表面积的目的 ${ }^{[31]}$ 。目前为止, 已经报道

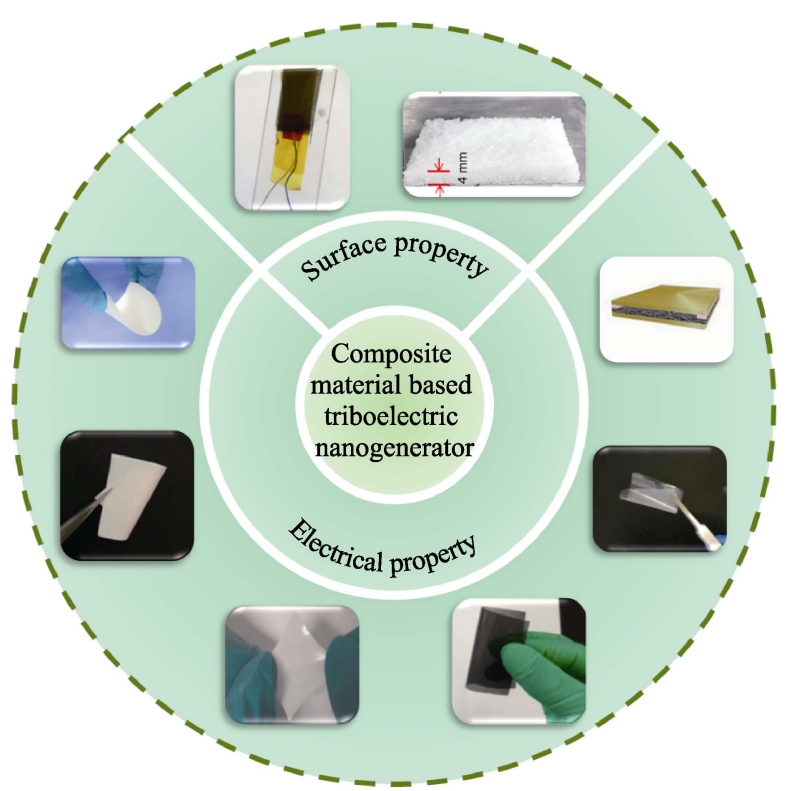

图 1 用于 TENG 的不同复合材料薄膜以及所制备的器件示 意图 ${ }^{[20-27]}$

Fig. 1 Schematic diagram of various composite films and devices for TENGs ${ }^{[20-27]}$ 
了各种优化摩擦层材料表面性能的技术，例如表面 氟硅烷化 ${ }^{[32]}$ 、硅模板倒膜 ${ }^{[33]}$ 、光刻 ${ }^{[34]}$ 、等离子体刻 蚀 ${ }^{[14]}$ 等, 这些方法要么稳定性差, 要么步骤繁琐需 要昂贵的设备及苛刻的实验条件, 并不适合大规模 的实际应用。而通过在高分子基底材料中添加微纳 米填料的方法来提高材料表面粗糙度，具有制备简 单、成本低廉、物理化学稳定性好以及可赋予器件 功能性等诸多优点。

基于以上理论, 印度理工学院的 Kaur 等 ${ }^{[20]}$ 在 PVDF 中添加纳米带状的还原氧化石墨烯(rGONRs), 制备了一种复合拱形单电极式 TENG，如图 2(a)所 示。这种 $\mathrm{rGONRs} / \mathrm{PVDF}$ 复合薄膜的平均表面粗糙 度远大于纯的 PVDF 膜, 在提高 TENG 输出性能上 起到了决定性作用。实验结果表明，基于这种复合 薄膜的 TENG 成功将其输出电压提高了 2 倍。 $\mathrm{NaCl}$ 颗粒以及 $\mathrm{ZnO}$ 纳米棒也都被作为提高高分子基体 介电层粗粮度的填料制备复合薄膜, 并有效提高了 器件的输出性能 ${ }^{[35-36]}$ 。另外, 无机填料也被添加到 三维微纳米结构的基体材料中, 以达到进一步增大 表面粗糙度的效果。上海师范大学的 Liu 等 ${ }^{[21]}$ 通过 牺牲模板法制备了基于海绵状 $\mathrm{TiO}_{2} / \mathrm{PDMS}$ 复合膜
的 TENG(CS-TENG)。由图 2(b)可见, $\mathrm{TiO}_{2}$ 纳米颗粒 分布在 PDMS 表面上, 进一步提高了网络结构 PDMS 的比表面积, 增大了其与铝䈃之间的有效接 触面积, 所产生的最大瞬时开路电压 $\left(V_{\mathrm{oc}}\right)$ 约为 $125 \mathrm{~V}$, 短路电流 $\left(I_{\mathrm{sc}}\right)$ 为 $2.9 \mu \mathrm{A}$, 最大转移电荷为 $40 \mathrm{nC}$ 。加 入 $\mathrm{TiO}_{2}$ 纳米颗粒不仅提高了 CS-TENG 输出性能, 还可作为光催化剂, 实现能量收集和自清洁的双重 功能, 这项研究拓展了 TENG 在光催化领域的应用。

另外，在构筑三维立体网络结构材料的技术中， 静电纺丝是一种简便高效的方法 ${ }^{[37-38]}$ 。而引入填料 为静电纺丝法制备多样的纳米纤维结构带来了更多 的可能性 ${ }^{[39]}$ 。例如, 西安交通大学 Chen 等 ${ }^{[40]}$ 通过 静电纺丝技术制成了一种具有分层微纳米结构的 $\mathrm{SiO}_{2} / \mathrm{P}(\mathrm{VDF}-\mathrm{TrFE})$ 复合薄膜并应用于可拉伸 TENG。如图 2(c)中 $\mathrm{SEM}$ 照片所示, $\mathrm{SiO}_{2}$ 纳米颗粒 作为填料充当纳米纤维的结点, 使薄膜呈现一种分 级结构的 3D 纳米纤维网络。对比于纯聚偏氟乙烯 共聚物 $(\mathrm{P}(\mathrm{VDF}-\mathrm{TrFE}))$ 纳米纤维薄膜, 复合薄膜的 表面粗糙度明显提高。经测试，其组装而成的 TENG 产生的最大瞬时输出电压约为 $170 \mathrm{~V}$, 电流密度为 $12.5 \mu \mathrm{A} / \mathrm{cm}^{2}$, 电荷转移密度为 $24 \mathrm{nC} / \mathrm{cm}^{2}$, 比纯

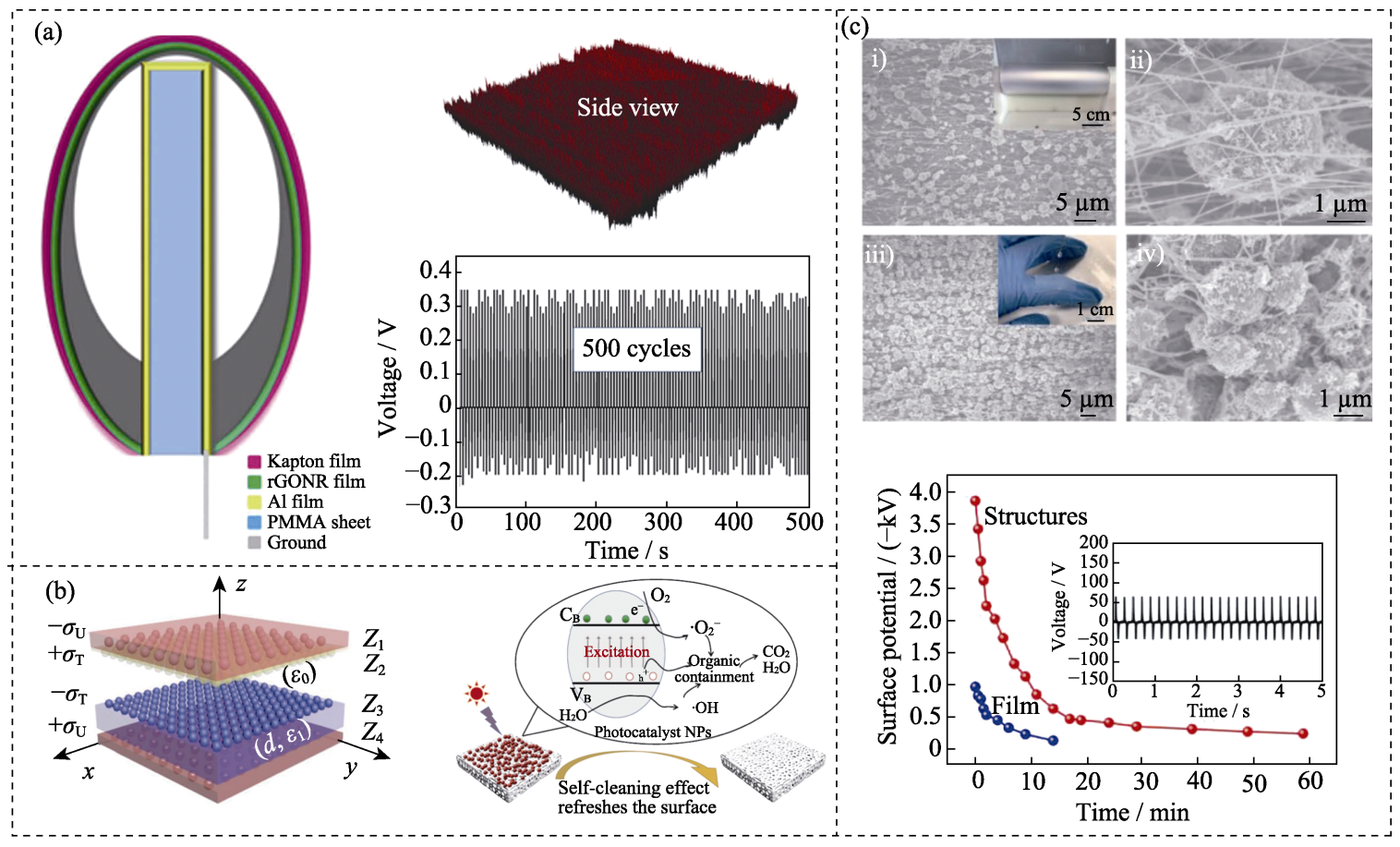

图 2 基于 $\mathrm{rGONRs/PVDF}$ 复合薄膜的 TENG 器件示意图、复合薄膜的 3D-AFM 图片以及器件工作 500 个循环的输出电压图 谱(a) ${ }^{[20]}$; 海绵状 $\mathrm{TiO}_{2} / \mathrm{PDMS}$ 的 TENG 器件示意图, $\mathrm{TiO}_{2} / \mathrm{PDMS}$ 薄膜光催化原理图(b) ${ }^{[21]}$; 静电纺丝制备分层结构 $\mathrm{SiO} / 2 \mathrm{P}(\mathrm{VDF}-\mathrm{TrFE})$

复合薄膜的 SEM 照片以及纯 P(VDF-TrFE)膜(蓝色)和 $\mathrm{SiO}_{2} / \mathrm{P}\left(\mathrm{VDF}-\mathrm{TrFE}\right.$ )复合薄膜(红色)的表面电势对比(c) ${ }^{[40]}$

Fig. 2 Schematic diagram of rGONRs /PVDF based TENG, 3D-AFM image of the rGONRs/PVDF thin film, and output voltage of the rGONRs/PVDF based TENG for 500 cycles $(\mathrm{a})^{[20]}$, schematic diagram of $\mathrm{TiO}_{2} / \mathrm{PDMS}$ sponge based TENG, schematic of organic containment degradation by photocatalyst $\mathrm{NPs}$ in $\mathrm{TiO}_{2} / \mathrm{PDMS}$ sponge $(\mathrm{b})^{[21]}$, and SEM images of hierarchical structures for $\mathrm{SiO}_{2} / \mathrm{P}(\mathrm{VDF}-\mathrm{TrFE})$ composite fabricated by electrospinning process, and the surface potentials of

pure $\mathrm{P}(\mathrm{VDF}-\mathrm{TrFE})$ film (blue) and $\mathrm{SiO}_{2} / \mathrm{P}(\mathrm{VDF}-\mathrm{TrFE})$ composite film (red) (c) ${ }^{[40]}$ (Colorful figures are available on website) 
$\mathrm{P}(\mathrm{VDF}-\mathrm{TrFE})$ 膜的输出电压提高了 3 倍。

上述工作证实了优化摩擦层材料表面性能对提 高 TENG 输出性能的积极作用。但是, 从输出性能 提升的数据来看, 仅从材料表面性能方面进行优化 所带来的性能提升比较有限。因此, 还需要从材料 的电学性能方面出发, 进一步提高 TENG 的输出功 率密度。

\section{2 优化材料电学性能}

摩擦层材料的电学性能决定了其在工作过程中 对电荷的捕获/储存能力, 因此优化摩擦层材料电学 性能也是一种有效提高表面电荷密度的方法。在现 有的研究中, 通常通过提高材料介电常数、增加电 荷捕获位点以及引入驻极体材料等方法对摩擦层材 料的电学性能进行优化, 使其能够更大程度地捕获/ 存储摩擦所产生的电荷, 减少器件工作过程中的电 荷损失, 从而达到提高摩擦层材料表面电荷密度的 目的 ${ }^{[41]}$ 。然而, 常用的摩擦电高分子聚合物材料的 介电性能和捕获电荷能力还远未达到实际应用的要 求。因此, 高介电常数、高电子捕获能力以及具有 驻极体性能的微纳米材料常常被研究者作为填料引 入高分子基底中优化其电学性能, 从而获得高输出 性能的 TENG。

\section{1 提高材料介电性能}

基于 TENG 的工作原理和垂直接触分离模式 TENG 的理论研究, 可以得出其表面电荷密度 $(\sigma)$ 与材料的介电性能之间的关系为 ${ }^{[42]}$ :

$$
\sigma=\frac{Q}{A}=\frac{C V_{\text {tri }}}{A}=\frac{\varepsilon_{0} \varepsilon_{\mathrm{r}} V_{\text {tri }}}{d} .
$$

其中, $Q$ 是摩擦层材料表面电荷量, $C$ 是电容, $V_{\mathrm{tr}}$ 是 摩擦电压, $\varepsilon_{0}$ 是真空介电常数, $\varepsilon_{\mathrm{r}}$ 是摩擦层材料的介 电系数, $A$ 是器件的有效接触面积, $d$ 是器件的厚度。 公式(1)表明, 提高摩擦层材料介电常数可有效增加 其工作时的表面电荷密度。

为了有效提高材料的介电常数, 很多研究者使 用了传统高介电性能钙钛矿相复合氧化物 (如 $\mathrm{BaTiO}_{3}{ }^{[43]}, \mathrm{SrTiO}_{3}{ }^{[44]}, \mathrm{ZnSnO}_{3}{ }^{[45]}$ )。上海交通大学 $\mathrm{Shi}$ 等 ${ }^{[22]}$ 展示了一种由多孔纤维素纸和 PDMS 组装而 成的 TENG。如图 3(a)所示, 通过 $\mathrm{BaTiO}_{3}$ 对纤维素 纸进行介电调制, 所得复合材料的介电常数(6.25)约 为纯纤维素纸介电常数(3.06)的两倍。掺杂之后的 TENG 最大输出功率提高了 3 倍, 达到 $141 \mu \mathrm{W}$, 并 成功应用于无线传感领域。Kang 等 ${ }^{[23]}$ 也采用 $\mathrm{BaTiO}_{3}$ 作为填料, 添加到 PVDF 基体材料中制备复
合薄膜。这种复合薄膜的介电常数随 $\mathrm{BaTiO}_{3}$ 体积分 数的增加而增大, 当体积分数达到 $11.25 \%$ 时, 介电 常数高达 25(图 3(b)), 可产生 $131 \mathrm{~V}$ 的峰值开路电 压和 $89 \mu \mathrm{C} \cdot \mathrm{m}^{-2}$ 的峰值短路电荷密度, 其峰值开路 电压比基于纯 PVDF 膜的 TENG 高 6.5 倍。重庆大 学 Wang 等 ${ }^{[24]}$ 则将 $\mathrm{ZnSnO}_{3}$ 纳米颗粒以不同比例分 散到 PDMS 弹性体中(图 3(c)), 当掺杂浓度为质量 分数 $6 \%$ 时, 器件可产生最大 $400 \mathrm{~V}, 28 \mu \mathrm{A}$ 的输出性 能, 峰值功率可达 $3 \mathrm{~mW}$, 与基于纯 PDMS 膜的 TENG 相比, 输出电流提高了 6.2 倍。新加坡国立 大学 Fang 等 ${ }^{[46]}$ 选择了 $\mathrm{CaCu}_{3} \mathrm{Ti}_{4} \mathrm{O}_{12}$ 颗粒作为填料嵌 入 PDMS 基质中。图 4(a)的结果表明, $\mathrm{CaCu}_{3} \mathrm{Ti}_{4} \mathrm{O}_{12}$ 的高介电性能显著提高了 PDMS 膜的介电常数和 表面电荷电势。他们通过优化填料重量比, 同时引 入表面图案化, 基于质量分数 $30 \% \mathrm{CaCu}_{3} \mathrm{Ti}_{4} \mathrm{O}_{12}$ (30CCTO)的图案化 PDMS 膜所制备的 TENG 产生 的最高输出功率密度达到 $9.6 \mathrm{~W} \cdot \mathrm{m}^{-2}$, 与基于纯的平 面 PDMS 膜的 TENG 相比, 输出功率密度提高了 10 倍(图 4(b))。通过这些工作之间的横向对比, 也进一 步验证了摩擦层材料的介电常数与其输出性能成 正比。

除了上述传统高介电无机填料, 金属有机框 架(MOF)材料也被应用于提高摩擦层材料的介电 性能 ${ }^{[47-48]}$ 。本课题组 ${ }^{[49]}$ 将一种氟化金属有机框架材 料(F-MOF) 作为填料掺入一系列高分子基体(包括 PDMS、PVDF、PTFE、硅橡胶等)中, 得到不同的 复合薄膜并组装成 TENG 器件。引入 F-MOF 在增 大薄膜的感应电荷量的同时有效提高了薄膜的介电 性能。有限元模拟(图 4(c)) 和实验结果表明, F-MOF 的优化能力适用于不同的高分子基体材料, 其中 PDMS@F-MOF 复合薄膜所组装的 TENG 峰值输出 功率密度达到 $52 \mu \mathrm{W} \cdot \mathrm{cm}^{-2}$, 比基于纯 PDMS 薄膜的 TENG 高出 11 倍(图 4(d))。这项研究表明 MOFs 材 料对优化摩擦层材料的电学性能也表现优异, 进一 步拓展了填料的选择范围。

\section{2 提高材料电荷捕获能力}

在介电摩擦层材料中添加导电或二维填料能够 为其提供丰富的电荷捕获位点、增加电荷移动路径, 从而捕获更多的电荷, 提高材料表面电荷密度, 是 得到高性能 TENG 的有效方法。重庆大学 $\mathrm{He}$ 等 ${ }^{[25]}$ 将尺寸为 $20 \sim 40 \mathrm{~nm}$ 且质量百分含量为 $3.0 \%$ 的石墨 颗粒(GP)与 PDMS 混合制备了基于PDMS@GP复合 薄膜的 TENG(CFNG), GP 良好的导电性能提高了复 合薄膜对电子的捕获能力, 因此 CFNG 的表面电荷 量提高了 $111.27 \%$, 可产生 $3.7 \mathrm{~W} \cdot \mathrm{m}^{-2}$ 的峰值输出功率 

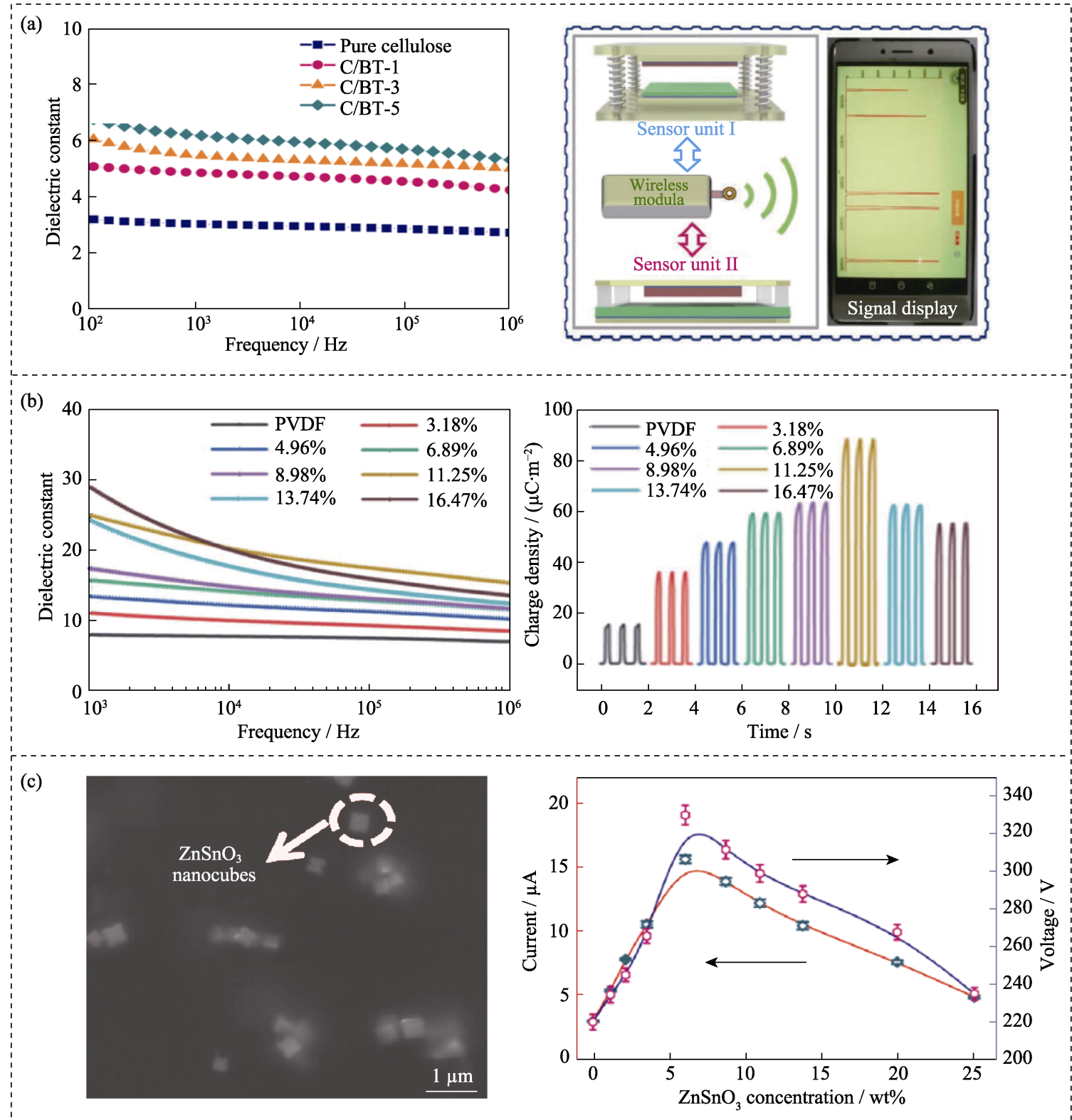

图 3 不同 $\mathrm{BaTiO}_{3}$ 含量的纤维素纸复合薄膜介电常数曲线(C/BT-1、C/BT-3 和 C/BT-5 样品中 $\mathrm{BaTiO}_{3}$ 质量含量分别为 $50 \%$ 、 $75 \%$ 和 $83.3 \%$ ) 以及器件用于无线通讯的示意图(a) ${ }^{[22]}$; 不同 $\mathrm{BaTiO}_{3}$ 含量的 $\mathrm{PVDF}$ 复合薄膜介电常数以及所组成器件表面电荷

密度对比图(b) ${ }^{[23]} ; \mathrm{ZnSnO}_{3} @ \mathrm{PDMS}$ 复合薄膜的表面扫描电镜照片及其不同含量时的输出电流与电压曲线(c) ${ }^{[24]}$

Fig. 3 Dielectric constants of the cellulose/ $\mathrm{BaTiO}_{3}$ aerogel paper with different $\mathrm{BaTiO}_{3}$ contents (the mass ratios of $\mathrm{BaTiO}_{3}$ in

C/BT-1, C/BT-3 and C/BT-5 were $50 \%, 75 \%$ and $83.3 \%$, respectively) and schematic image of wireless application of the

TENG(a) ${ }^{[22]}$, dielectric constants and charge densities of the $\mathrm{BaTiO}_{3} / \mathrm{PVDF}$ nanocomposite films with different $\mathrm{BaTiO}_{3}$ volume fractions (b) ${ }^{[23]}$, and SEM image of $\mathrm{ZnSnO}_{3} @$ PDMS composite film, and output currents and voltages of the corresponding TENGs with different $\mathrm{ZnSnO}_{3}$ contents (c) ${ }^{[24]}$ (Colorful figures are available on website)

密度, 与未添加 GP 的 TENG 相比, 其输出功率密度 提高了 2.6 倍(图 5(a))。Harnchana 等 ${ }^{[50]}$ 开发出了一 种PDMS@GO@SDS 复合薄膜作为高性能摩擦层材 料, 如图 5(b)所示。嵌入的 $\mathrm{GO}$ 在复合膜中充当电 荷俘获位点, 有效增强了电荷捕获能力。同时，阴离 子表面活性剂十二烷基硫酸钠(SDS)和 GO 还协同 增强了负电荷密度。这些改进与大接触面积的多孔 结构相结合, 进一步提高了PDMS@GO@SDS 复合
薄膜的输出性能, 多孔PDMS@GO@SDS 复合薄膜 可以提供 $438 \mathrm{~V}$ 和 $11 \mu \mathrm{A} \cdot \mathrm{cm}^{-2}$ 的峰值输出电压和电流, 是平面 PDMS 膜的 3 倍, 能够直接点亮 90 个 LED。

近年来，除石墨烯之外的新型二维材料高频率 地出现在各个应用领域中, 研究者们也将这些材料 应用于 $\mathrm{TENG}$ 领域。 Kim 等 ${ }^{[11]}$ 提出了一种将铁电聚 合物与 $\mathrm{MoS}_{2}$ 掺杂制备复合材料以有效提高 TENG 输出电流密度的方法。他们将二维 $\mathrm{MoS}_{2}$ 分别与 


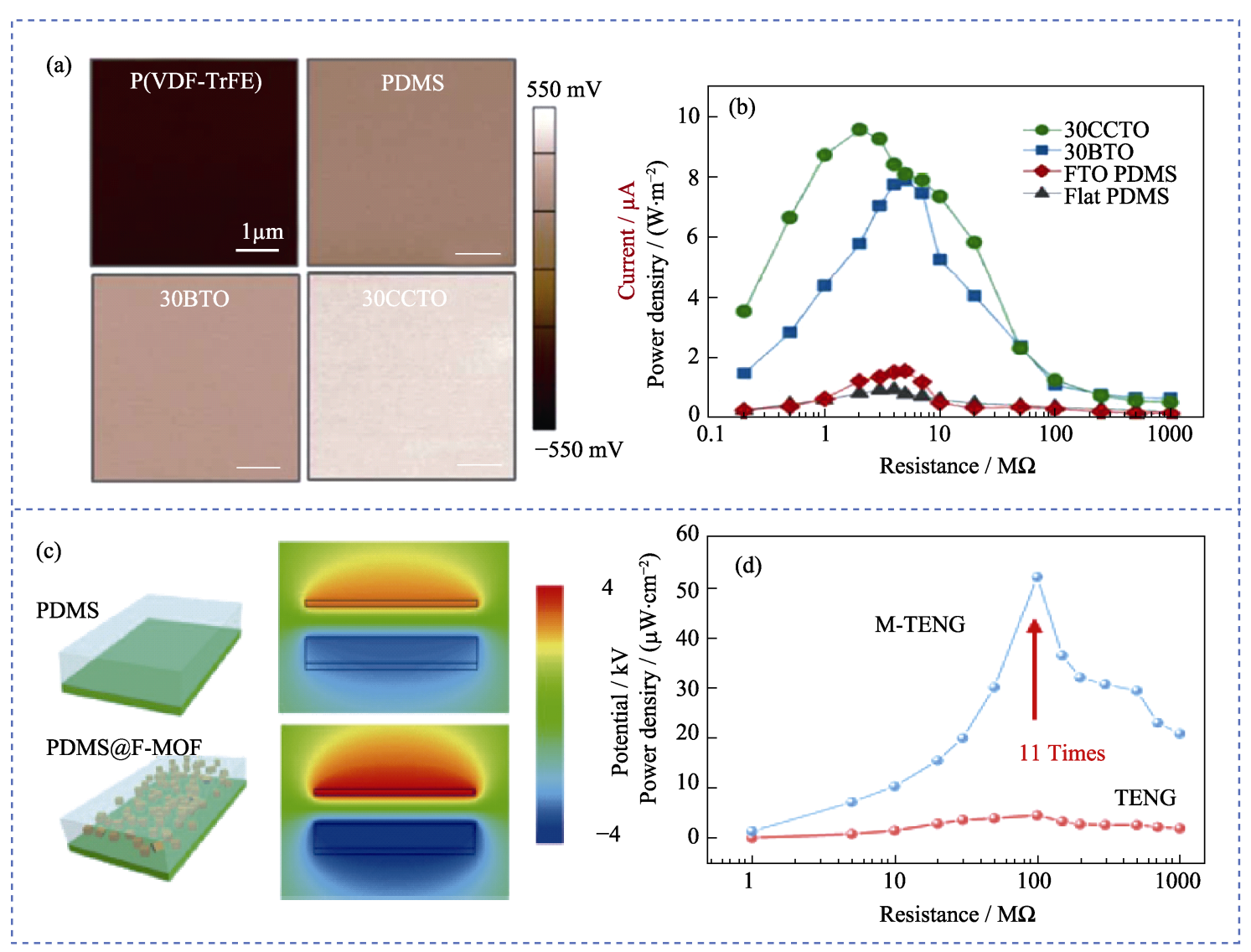

图 4 P(VDF-TrFE)、PDMS、PDMS-30wt\% BTO(30BTO)和 PDMS-30wt\% CCTO (30CCTO)薄膜的开尔文 探针力显微镜(KPFM)图像(a)以及其输出功率密度(b) ${ }^{[46]}$; 基于 PDMS 以及 PDMS@F-MOF 薄膜器件的电场分布(有限元模拟)(c)及其实际输出功率密度 $(\mathrm{d})^{[49]}$

Fig. 4 KPFM images (a) and power densities (b) of P(VDF-TrFE), PDMS, 30BTO and 30CCTO films ${ }^{[46]}$, electrical field distributions (finite-element simulation) (c) and power densities as a function of the external resistance $(d)$ of the devices based on PDMS and PDMS@F-MOF ${ }^{[49]}$

Nylon-11 和 P(VDF-TrFE)混合制备复合薄膜, 以显 著提高各摩擦层的表面电荷密度。图 5(c)展示了三 种 TENG 的表面电荷密度, 这三种器件分别是: 纯 Nylon-11 和 P(VDF-TrFE)组成的器件, Nylon-11@ $\mathrm{MoS}_{2}$ 和 P(VDF-TrFE)@ $\mathrm{MoS}_{2}$ 复合薄膜组成的器件的未极 化状态和极化状态。所得基于复合材料的 TENG 在 极化状态下最终可产生 $145 \mathrm{~V}, 350 \mu \mathrm{A} \cdot \mathrm{cm}^{-2}$ 的峰值 输出电压和电流, 以及 $50 \mathrm{~mW} \cdot \mathrm{cm}^{-2}$ 的峰值输出功 率密度, 与未掺杂的 TENG 相比, 功率密度提高了 8 倍。由此发现, 掺杂二维 $\mathrm{MoS}_{2}$ 是提高聚合物表面 电势的有效方法。Wen 等 ${ }^{[26]}$ 制成基于 PVDF 和单层 二氧化钛(TOML)的纳米复合薄膜, 设计和制备了一 种柔性透明的 TENG(图 5(d))。与基于纯 PVDF 膜的 TENG 相比, 基于含质量百分比 $1.5 \% \mathrm{TOML}$ 的 PVDF/TOML 复合材料的 TENG 可产生 $52.8 \mathrm{~V}$ 和 $5.69 \mu \mathrm{A} \cdot \mathrm{cm}^{-2}$ 的 峰值输出性能, 峰值输出功率密度提高了 50 倍。 TENG 输出性能的增强主要得益于 TOML 较大的比 表面积, 提供了较多的电荷捕获位点, 很大程度上 减少了薄膜表面摩擦感应电荷的损失。新加坡南洋
理工大学 Cui 等 ${ }^{[27]}$ 将二维黑磷和纤维素纳米纤维 $(\mathrm{CNF})$ 混合后通过抽滤的方法制备了一种透明且柔 性的复合纸基 TENG(图 5(e))。黑磷纳米带均匀地分 散在 $\mathrm{CNF}$ 基体中, 为薄膜提供了极为丰富的电荷俘 获位点, 极大地提高了 TENG 的表面电荷密度。 $\mathrm{CNF}$ /黑磷基 TENG 实现了 $5.2 \mathrm{~V}$ 的开路电压(图 5(f)) 和 $1.8 \mu \mathrm{A} \cdot \mathrm{cm}^{-2}$ 的电流密度, 峰值功率密度比基于纯 CNF 的 TENG 高 46 倍。由此可见, 由于这些新型 二维填料具有较大的比表面积, 暴露了更多的活性 位点, 进而能够捕获和储存更多的电荷, 相比于零 维与一维材料, 其对于 TENG 的输出功率密度的提 升程度更大。因此, 需要研究更多的二维活性材料, 有望突破性提高 TENG 的输出性能。

\section{3 引入驻极体填料}

驻极体 (也称为永电体), 是指能够“长期”存储 过剩“真实”电荷或/和保持“取向”电偶极子的电介 质。常用驻极体材料主要分为两类: 第一类是以氧 化硅 $\left(\mathrm{SiO}_{2}\right)$ 为代表的无机驻极体; 第二类是聚合物 有机驻极体，例如全氟乙烯丙烯共聚物(FEP)、聚四 

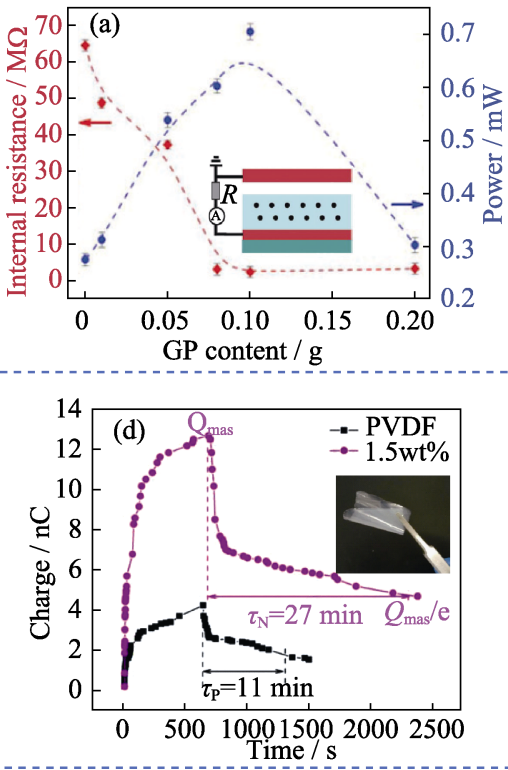
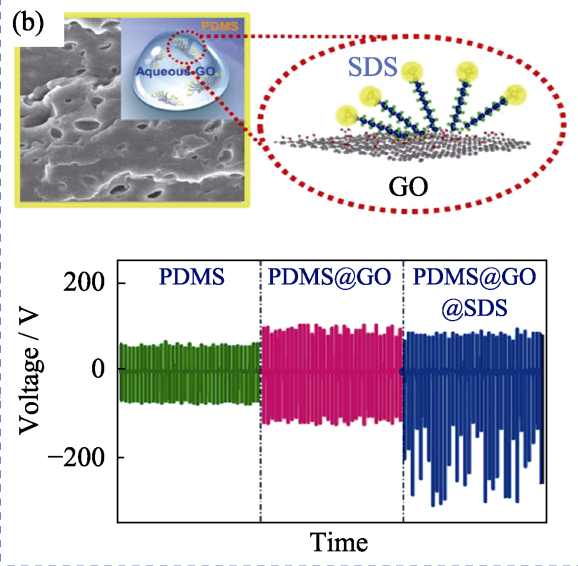

(e)

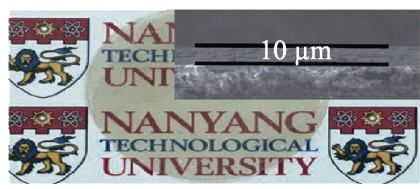

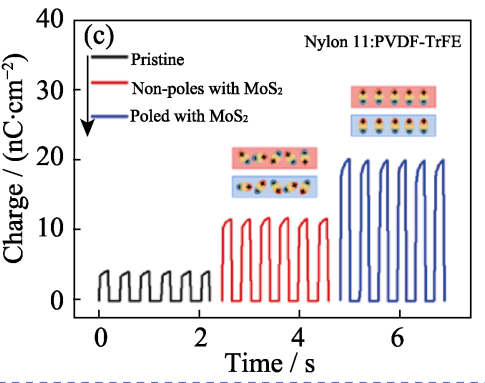

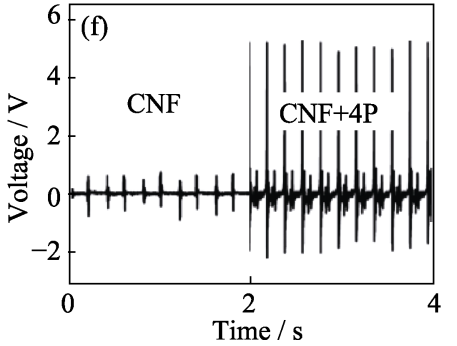

图 5 不同 GP 含量的复合薄膜的 TENG 内阻以及输出功率, 插图为器件结构示意图(a) ${ }^{[25]}$; PDMS@GO@SDS 复合薄膜的示意图, 基于不同薄膜的 TENG 输出电压对比图(b) ${ }^{[50]}$; 纯 Nylon-11 和 PVDF-TrFE(黑色), Nylon-11@ $\mathrm{MoS}_{2}$ 和 P(VDF-TrFE)@MoS 2 复合薄膜 组成的器件的未极化状态(红色)和极化状态(蓝色)的 TENG 的电荷密度比较(c) ${ }^{[51]}$; 纯 PVDF 薄膜和 PVDF/TOML 复合膜的 TENG 表面电荷对比图, 以及 PVDF/TOML 复合膜照片(d) ${ }^{[26]} ; \mathrm{CNF} /$ 黑磷复合薄膜的照片 (e), 及单纯 $\mathrm{CNF}$ 与 $\mathrm{CNF} /$ 黑磷复合薄膜所制备的 TENG 输出电压对比图(f) ${ }^{[27]}$

Fig. 5 Internal resistances and output powers of PDMS@GPs composite membranes with different GP contents with inset showing structural schematic of corresponding TENG device (a ${ }^{[25]}$, schematic image of PDMS@GO@SDS composite film and the output voltages of different TENGs $(b)^{[50]}$, comparison of charge densities for TENGs : pure Nylon-11 and PVDF-TrFE (black), Nylon-11@MoS $\mathrm{M}_{2}$ and $\mathrm{P}(\mathrm{VDF}-\mathrm{TrFE}) @ \mathrm{MoS}_{2}$ composite films in non-poled state (red) and poled state (blue) (c) ${ }^{[51]}$, comparison of charges for PVDF and PVDF/TOML nanocomposite films based TENGs with inset showing the picture of PVDF/TOML nanocomposite film (d) ${ }^{[26]}$, optical image of the $\mathrm{CNF}$ /phosphorene hybrid paper (e), and the comparison of voltages between pure CNF based TENG and CNF/ phosphorene hybrid paper based TENG (f $)^{[27]}$

氟乙烯(PTFE)、聚偏氟乙烯(PVDF)以及聚对苯二甲 酸乙二醇酯(PET) 等 ${ }^{[52]}$ 。由于驻极体有优良的储存 电荷能力, 因此被广泛应用于能源器件中 ${ }^{[53]}$ 。在摩 擦纳米发电机领域, 大量研究采用上述驻极体高分 子薄膜作为介电摩擦层, 取得了很好的效果 ${ }^{[54]}$ 。本 文主要介绍无机驻极体作为填料的复合薄膜在 TENG 中的应用。

如图 6(a)所示, 本课题组 ${ }^{[55]}$ 将改性的疏水 $\mathrm{SiO}_{2}$ 纳米微球加入聚合物基底 PVDF 中, 通过静电纺丝 过程中高电场作用将其极化, 制备了复合纳米纺丝 膜。通过表面电势测试发现, 加入 $\mathrm{SiO}_{2}$ 驻极体有效 降低了复合薄膜表面电荷的损失, $180 \mathrm{~min}$ 后表面电 势仍保持约 40\%, 而纯基底薄膜表面电势已经降低 为 0 (图 6(b))。这种驻极体特性提高了器件的电荷密 度, 所制备的 TENG 器件最高功率密度提高了约 5.3 倍。同样, 东华大学 Xiao 课题组 ${ }^{[56]}$ 将 $\mathrm{SiO}_{2}$ 驻极体 添加到一种热塑性纳米纤维膜中, 将其峰值电压、 电流分别提高到 $144.9 \mathrm{~V}$ 和 $23.7 \mu \mathrm{A}$ 。在 TENG 领域 中应用的无机驻极体种类单一, 且对器件性能提升 有限。这是由于单一 $\mathrm{SiO}_{2}$ 驻极体存在长期稳定性差
的问题，荷兰特文特大学的 Mendel 等 ${ }^{[57]}$ 发现制备 氧化物一氟化聚合物复合驻极体能够有效改善两种 类型驻极体各自的缺陷。他们制备了 $\mathrm{SiO}_{2}-\mathrm{FP}(\mathrm{Teflon}$ AF 1600)复合驻极体, 并通过电晕充电的方法将载 流子注入复合薄膜中测试了其表面电荷密度以及电 荷损失速率，结果表明这种复合驻极体能够将载流 子深度储存在聚合物与氧化物界面中, 在高温条件 下依然保持很大的电荷密度(图 6(c, d))。这种有机-无 机复合驻极体在能源器件领域拥有巨大的应用潜力, 而其在 TENG 领域中还鲜有研究。

\section{3 结论与展望}

总体来说, 多种复合材料体系在 TENG 中的应 用切实提高了其输出性能。表 1 总结了本文提及的 复合材料体系的一些参数, 从中可见, 当填料仅用 于增大摩擦层表面粗糙度时, 其对输出性能的提高 程度有限; 而相比于优化材料表面性能, 提高材料 电学性能对器件输出性能的影响更为显著。尤其是 引入一些功能型二维材料, 将 TENG 的输出功率密 


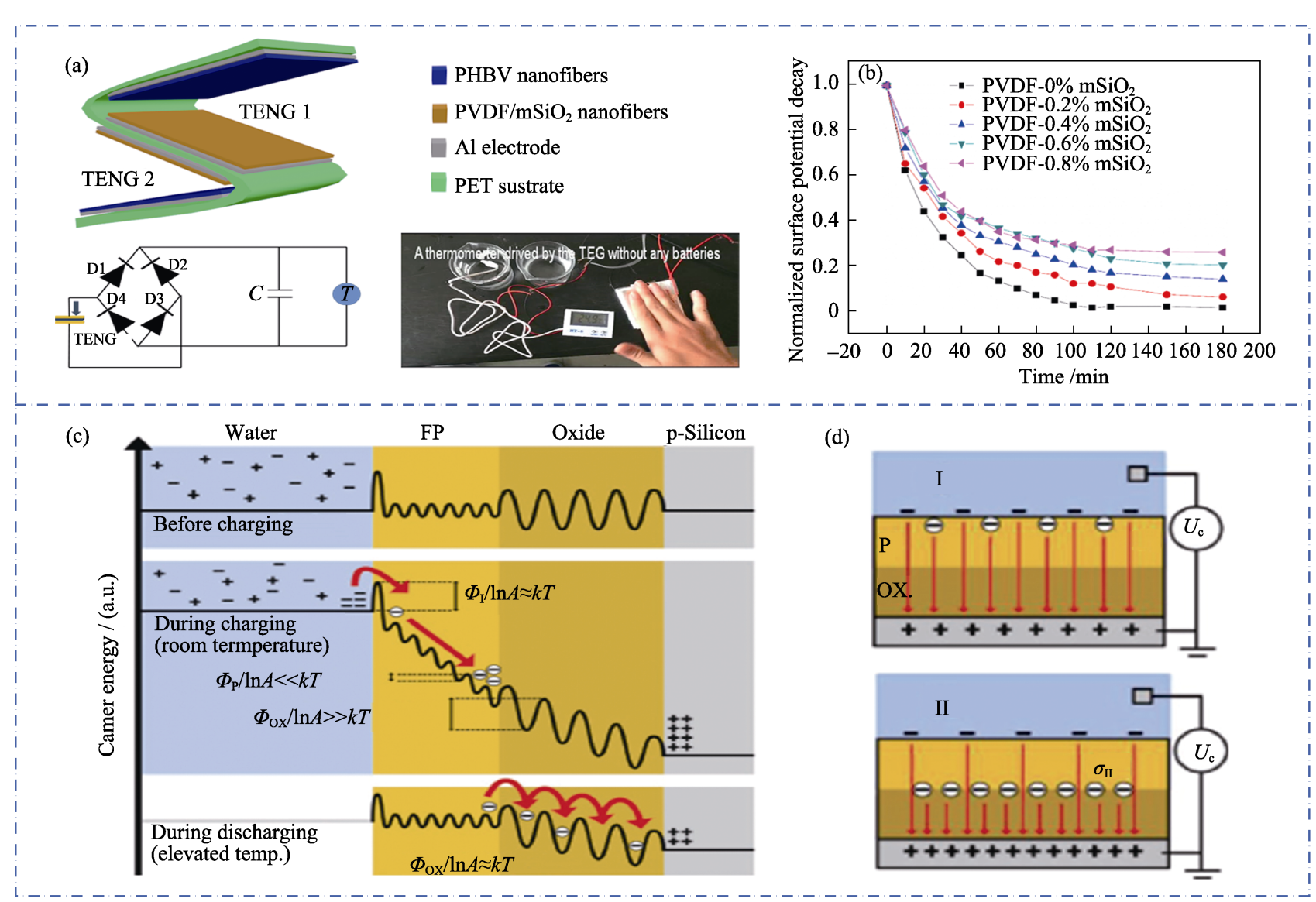

图 6 书形 TENG 的制备过程示意图(a), 具有不同 $\mathrm{mSiO}_{2}$ 浓度的 $\mathrm{PVDF} / \mathrm{mSiO}_{2}$ 纳米纤维的表面电势衰减曲线 $(\mathrm{b})^{[55]} ; \mathrm{SiO}_{2}-\mathrm{FP}$

在充电前、充电过程中电荷载流子的能量分布图, 以及高温下的表面电势衰减(c); 充电过程中在注入

电荷位于水-FP 界面(I), 注入电荷位于 FP-氧化物界面(II)两种竞争情况下的电场分布(d) ${ }^{[57]}$

Fig. 6 Illustration of the fabrication process of a book-shaped TENG (a), normalized surface potential decay of $\mathrm{PVDF} / \mathrm{mSiO}{ }_{2}$ nanofibers with different concentrations of $\mathrm{mSiO}_{2}(\mathrm{~b})^{[55]}$; energy landscapes for (injected) charge carriers for $\mathrm{SiO}_{2}-\mathrm{FP}$ before and

during charging, and surface potential decays at elevated temperatures (c), electric field distributions during charging for two competing scenarios with injected charge residing at the aqueous-FP interface (I), and injected charge residing at the FP-oxide interface (II) (d) ${ }^{[57]}$

表 1 用于 TENG 复合材料的填料种类

Table 1 Fillers used in composite materials for TENGs

\begin{tabular}{|c|c|c|c|c|c|c|c|}
\hline \multirow{2}{*}{ Filler } & \multirow{2}{*}{ Matrix } & \multicolumn{2}{|c|}{ Optimal fillers ratio } & \multirow{2}{*}{$\begin{array}{c}\text { Dielectric } \\
\text { constant }\end{array}$} & \multirow{2}{*}{ Shape/Size } & \multirow{2}{*}{$\begin{array}{l}\text { Increased percentage } \\
\text { of output } / \%\end{array}$} & \multirow{2}{*}{ Ref. } \\
\hline & & $\mathrm{wt} \%$ & vol\% & & & & \\
\hline rGONRs & PVDF & 97 & - & - & Nanoribbon & 200 (Voltage) & {$[20]$} \\
\hline $\mathrm{TiO}_{2}$ & PDMS & 0.05 & - & - & Nanopaticle & - & {$[21]$} \\
\hline $\mathrm{SiO}_{2}$ & $\mathrm{P}(\mathrm{VDF}-\mathrm{TrFE})$ & 30 & - & - & Nanopaticle $(D=10-20 \mathrm{~nm})$ & 300 (Voltage) & {$[40]$} \\
\hline $\mathrm{BaTiO}_{3}$ & Cellulose paper & 16.7 & - & 6.25 & Nanopaticle $(D=200 \mathrm{~nm})$ & 300 (Power) & {$[22]$} \\
\hline $\mathrm{BaTiO}_{3}$ & PVDF & - & 11.25 & 25 & Nanopaticle $(D=100 \mathrm{~nm})$ & 650 (Voltage) & {$[23]$} \\
\hline $\mathrm{ZnSnO}_{3}$ & PDMS & 6 & - & - & Nanocube & 620 (Current) & {$[24]$} \\
\hline $\mathrm{CaCu}_{3} \mathrm{Ti}_{4} \mathrm{O}_{12}$ & PDMS & 30 & - & - & Nanopaticle & 1000 (Power) & [46] \\
\hline KUAST-8 & PDMS & 0.5 & - & 4.23 & Nanopaticle & 1100 (Power) & [49] \\
\hline Graphite particle & PDMS & 3 & - & 3 & Nanopaticle $(D=20-40 \mathrm{~nm})$ & 260 (Power) & {$[25]$} \\
\hline GO & PDMS & - & 16.7 & - & Nanosheet & 300 (Voltage) & {$[50]$} \\
\hline $\mathrm{MoS}_{2}$ & Nylon-11/P(VDF-TrFE) & - & - & - & Nanosheet & 800 (Power) & {$[51]$} \\
\hline Monolayer titania & PVDF & 1.5 & - & 11.51 & Thickness $=1.2 \mathrm{~nm}$ & 5000 (Power) & {$[26]$} \\
\hline Phosphorene & $\mathrm{CNF}$ & 0.2 & - & - & Nanosheet & 4600 (Power) & {$[27]$} \\
\hline Hydrophobic $\mathrm{SiO}_{2}$ & PVDF & 0.8 & - & - & Nanopaticle & 530 (Power) & {$[55]$} \\
\hline $\mathrm{SiO}_{2}$ & $\begin{array}{l}\text { Thermoplastic nanofiber } \\
\text { membranes }\end{array}$ & - & - & - & Nanopaticle & - & {$[57]$} \\
\hline
\end{tabular}


度提高了数十倍之多。不仅如此, 复合材料的应用 极大拓展了 TENG 器件的应用范围, 由原本的能源 收集到自供能传感、光催化、压电效应、铁磁性及 铁电性等领域。尽管该领域的研究已经获得了丰硕 的成果, 但是 TENG 的输出性能依然停留在毫瓦级 别, 还存在很大的提升空间, 机遇与挑战并存, 基 础理论研究及产业化发展仍有诸多工作需要进一步 开展:

1)填料的种类需要进一步丰富: 从以上诸多研 究成果来看, TENG 的输出性能随着各种新填料的 引入不断提高。这些填料大都只能从表面性能或电 学性能单一方面来改善复合材料的摩擦电性能, 虽 然已经有部分填料如 F-MOF、TOML 等能够同时从 两方面提高性能, 但这类研究还处于初级阶段。因 此，寻找或设计更多的多功能填料，如有机金属框 架材料、钙铁矿材料、新型二维材料、液态金属等, 并 将其更好地应用在 TENG 领域, 还有待进一步研究。

2)复合材料体系有待拓展: 目前的复合材料体 系大多是以高分子聚合物作为基体材料, 固态微纳 米低维材料作为填料的固-固体系。以高介电常数液 体为填料的固-液体系虽然有一定优势但相关研究 较少。这些体系中填料的种类也大都只有一种。因 此, 固-液体系、固-气体系以及两种甚至多种填料 体系的研究需要进一步展开。

3)TENG 器件的实际应用问题有待解决: 目前 国内外研究的复合材料体系都能够有效提高 TENG 的输出性能。然而研究者们大多比较注重优化机理 上的理论研究, 往往忽略了器件的产业化应用问 题。缺少针对不同应用场景需求的材料体系的构建 和性能测试。因此，复合材料基 TENG 的产业化应 用研究仍需加强。

\section{参考文献:}

[1] FAN F R, TIAN Z Q, WANG Z L. Flexible triboelectric generator. Nano Energy, 2012, 1(2): 328-334.

[2] WANG J, ZHANG H, XIE Y, et al. Smart network node based on hybrid nanogenerator for self-powered multifunctional sensing. Nano Energy, 2017, 33: 418-426.

[3] ZHANG L, ZHANG B, CHEN J, et al. Lawn structured triboelectric nanogenerators for scavenging sweeping wind energy on rooftops. Adv. Mater., 2016, 28(8): 1650-1656.

[4] CHEN B, YANG Y, WANG Z L. Scavenging wind energy by triboelectric nanogenerators. Adv. Energy Mater., 2018, 8(10): 1702649.

[5] CHEN B D, TANG W, HE C, et al. Water wave energy harvesting and self-powered liquid-surface fluctuation sensing based on bionic-jellyfish triboelectric nanogenerator. Mater. Today, 2018, 21(1): 88-97.

[6] WANG X, WEN Z, GUO H, et al. Fully packaged blue energy harvester by hybridizing a rolling triboelectric nanogenerator and an electromagnetic generator. ACS Nano, 2016, 10(12): 11369-11376.

[7] MA Y F, TONG Z M, WANG M, et al. Triboelectric nanogenerator based on graphene forest electrodes. J. Inorg. Mater., 2019, 34(8): 839-843.

[8] FENG X, ZHANG Y, KANG L, et al. Integrated energy storage system based on triboelectric nanogenerator in electronic devices. Front. Chem. Sci. Eng., 2021, 15(2): 238-250.

[9] MAMUN M A A, YUCE M R. Recent progress in nanomaterial enabled chemical sensors for wearable environmental monitoring applications. Adv. Funct. Mater, 2020, 30(51): 2005703.

[10] ZHU M, HE T, LEE C. Technologies toward next generation human machine interfaces: from machine learning enhanced tactile sensing to neuromorphic sensory systems. Appl. Phys. Rev., 2020, 7(3): 031305.

[11] CHEN A, ZHANG C, ZHU G, et al. Polymer materials for high-performance triboelectric nanogenerators. $A d v$. Sci., 2020, 7(14): 2000186.

[12] BURGO T A, DUCATI T R, FRANCISCO K R, et al. Triboelectricity: macroscopic charge patterns formed by self-arraying ions on polymer surfaces. Langmuir, 2012, 28(19): 7407-7416.

[13] ZHU G, ZHOU Y S, BAI P, et al. A shape-adaptive thin-film-based approach for $50 \%$ high-efficiency energy generation through micro-grating sliding electrification. Adv. Mater, 2014, 26(23): 3788-3796.

[14] XIE Y, WANG S, NIU S, et al. Grating-structured freestanding triboelectric-layer nanogenerator for harvesting mechanical energy at $85 \%$ total conversion efficiency. Adv. Mater, 2014, 26(38): 6599-6607.

[15] DENG W, ZHANG B, JIN L, et al. Enhanced performance of ZnO microballoon arrays for a triboelectric nanogenerator. Nanotechnology, 2017, 28(13): 135401.

[16] SU L, ZHAO Z X, LI H Y, et al. High-performance organolead halide perovskite-based self-powered triboelectric photodetector. ACS Nano, 2015, 9(11): 11310-11316.

[17] YUE X, XI Y, HU C, et al. Enhanced output-power of nanogenerator by modifying PDMS film with lateral $\mathrm{ZnO}$ nanotubes and $\mathrm{Ag}$ nanowires. RSC Adv., 2015, 5(41): 32566-32571.

[18] WU C, KIM T W, PARK J H, et al. Enhanced triboelectric nanogenerators based on $\mathrm{MoS}_{2}$ monolayer nanocomposites acting as electron-acceptor layers. ACS Nano, 2017, 11(8): 8356-8363.

[19] LI G Z, WANG G G, YE D M, et al. High-performance transparent and flexible triboelectric nanogenerators based on PDMS-PTFE composite films. Adv. Electron. Mater, 2019, 5(4): 1800846.

[20] KAUR N, BAHADUR J, PANWAR V, et al. Effective energy harvesting from a single electrode based triboelectric nanogenerator. Sci. Rep., 2016, 6(1): 38835.

[21] LIU H, FENG Y, SHAO J, et al. Self-cleaning triboelectric nanogenerator based on $\mathrm{TiO}_{2}$ photocatalysis. Nano Energy, 2020, 70: 104499 .

[22] SHI K, ZOU H, SUN B, et al. Dielectric modulated cellulose paper/PDMS-based triboelectric nanogenerators for wireless transmission and electropolymerization applications. Adv. Funct. Mater, 2019, 30(4): 1904536.

[23] KANG X, PAN C, CHEN Y, et al. Boosting performances of triboelectric nanogenerators by optimizing dielectric properties and thickness of electrification layer. RSC Adv., 2020, 10(30): 17752-17759.

[24] WANG G, XI Y, XUAN H, et al. Hybrid nanogenerators based on triboelectrification of a dielectric composite made of lead-free $\mathrm{ZnSnO}_{3}$ nanocubes. Nano Energy, 2015, 18: 28-36.

[25] HE X, GUO H, YUE X, et al. Improving energy conversion efficiency for triboelectric nanogenerator with capacitor structure by maximizing surface charge density. Nanoscale, 2015, 7(5): 
$1896-1903$.

[26] WEN R, GUO J, YU A, et al. Remarkably enhanced triboelectric nanogenerator based on flexible and transparent monolayer titania nanocomposite. Nano Energy, 2018, 50: 140-147.

[27] CUI P, PARIDA K, LIN M F, et al. Transparent, flexible cellulose nanofibril-phosphorene hybrid paper as triboelectric nanogenerator. Adv. Mater. Interfaces, 2017, 4(22): 1700651.

[28] DIAZ A F, FELIX-NAVARRO R M. A semi-quantitative triboelectric series for polymeric materials: the influence of chemical structure and properties. J. Electrost., 2004, 62(4): 277-290.

[29] WANG Z L, WANG A C. On the origin of contact-electrification. Mater. Today, 2019, 30: 34-51.

[30] WU C, WANG A C, DING W, et al. Triboelectric nanogenerator: a foundation of the energy for the new era. Adv. Energy Mater., 2019, 9(1): 1802906.

[31] BAYTEKIN H T, PATASHINSKI A Z, BRANICKI M, et al. The mosaic of surface charge in contact electrification. Science, 2011, 333(6040): 308-12.

[32] GUO Y, LI K, HOU C, et al. Fluoroalkylsilane-modified textile-based personal energy management device for multifunctional wearable applications. ACS Appl. Mater. Interfaces, 2016, 8(7): 4676-4683.

[33] CHEN X, PU X, JIANG T, et al. Tunable optical modulator by coupling a triboelectric nanogenerator and a dielectric elastomer. Adv. Funct. Mater., 2017, 27(1): 1603788.

[34] KIM D, PARK S J, JEON S B, et al. A triboelectric sponge fabricated from a cube sugar template by 3D soft lithography for superhydrophobicity and elasticity. Adv. Electron. Mater., 2016, 2(4): 1500331

[35] SRIPHAN S, VITTAYAKORN N. Facile roughness fabrications and their roughness effects on electrical outputs of the triboelectric nanogenerator. Smart Mater. Struct., 2018, 27(10): 105026.

[36] SINGH H H, KHARE N. Improved performance of ferroelectric nanocomposite flexible film based triboelectric nanogenerator by controlling surface morphology, polarizability, and hydrophobicity. Energy, 2019, 178: 765-771.

[37] GONG W, HOU C, GUO Y, et al. A wearable, fibroid, self-powered active kinematic sensor based on stretchable sheath-core structural triboelectric fibers. Nano Energy, 2017, 39: 673-683.

[38] YANG W, GONG W, HOU C, et al. All-fiber tribo-ferroelectric synergistic electronics with high thermal-moisture stability and comfortability. Nat. Commun., 2019, 10: 5541.

[39] GONG W, HOU C, ZHOU J, et al. Continuous and scalable manufacture of amphibious energy yarns and textiles. Nat. Commun., 2019, 10(1): 868

[40] CHEN X, XIONG J, PARIDA K, et al. Transparent and stretchable bimodal triboelectric nanogenerators with hierarchical micronanostructures for mechanical and water energy harvesting. Nano Energy, 2019, 64: 103904.

[41] SEUNG W, YOON H J, KIM T Y, et al. Boosting power-generating performance of triboelectric nanogenerators via artificial control of ferroelectric polarization and dielectric properties. Adv. Energy Mater, 2017, 7(2): 1600988

[42] NIU S M, WANG Z L. Theoretical systems of triboelectric nanogenerators. Nano Energy, 2015, 14: 161-192.
[43] SHAO Y, FENG C P, DENG B W, et al. Facile method to enhance output performance of bacterial cellulose nanofiber based triboelectric nanogenerator by controlling micro-nano structure and dielectric constant. Nano Energy, 2019, 62: 620-627.

[44] CHEN J, GUO H, HE X, et al. Enhancing performance of triboelectric nanogenerator by filling high dielectric nanoparticles into sponge PDMS film. ACS Appl. Mater. Interfaces, 2016, 8(1): 736-744.

[45] LEE K Y, KIM D, LEE J H, et al. Unidirectional high-power generation via stress-induced dipole alignment from $\mathrm{ZnSnO}_{3}$ nanocubes/polymer hybrid piezoelectric nanogenerator. Adv. Funct. Mater, 2014, 24(1): 37-43.

[46] FANG Z, CHAN K H, LU X, et al. Surface texturing and dielectric property tuning toward boosting of triboelectric nanogenerator performance. J. Mater. Chem. A, 2018, 6(1): 52-57.

[47] WEN R, GUO J, YU A, et al. Humidity-resistive triboelectric nanogenerator fabricated using metal organic framework composite. Adv. Funct. Mater, 2019, 29(20): 1807655.

[48] KHANDELWAL G, CHANDRASEKHAR A, MARIA JOSEPH RAJ N P, et al. Metal-organic framework: a novel material for triboelectric nanogenerator-based self-powered sensors and systems. Adv. Energy Mater, 2019, 9(14): 1803581.

[49] GUO Y, CAO Y, CHEN Z, et al. Fluorinated metal-organic framework as bifunctional filler toward highly improving output performance of triboelectric nanogenerators. Nano Energy, 2020, 70: 104517.

[50] HARNCHANA V, NGOC H V, HE W, et al. Enhanced power output of a triboelectric nanogenerator using poly(dimethylsiloxane) modified with graphene oxide and sodium dodecyl sulfate. $A C S$ Appl. Mater. Interfaces, 2018, 10(30): 25263-25272.

[51] KIM M, PARK D, ALAM M M, et al. Remarkable output power density enhancement of triboelectric nanogenerators via polarized ferroelectric polymers and bulk $\mathrm{MoS}_{2}$ composites. ACS Nano, 2019, 13(4): 4640-4646.

[52] TAO K, YI H, YANG Y, et al. Origami-inspired electret-based triboelectric generator for biomechanical and ocean wave energy harvesting. Nano Energy, 2020, 67: 104197.

[53] HINCHET R, GHAFFARINEJAD A, LU Y, et al. Understanding and modeling of triboelectric-electret nanogenerator. Nano Energy, 2018, 47: 401-409.

[54] LIU L, TANG W, WANG Z L. Inductively-coupled-plasma-induced electret enhancement for triboelectric nanogenerators. Nanotechnology, 2017, 28(3): 035405.

[55] HUANG T, YU H, WANG $\mathrm{H}$, et al. Hydrophobic $\mathrm{SiO}_{2}$ electret enhances the performance of poly(vinylidene fluoride) nanofiberbased triboelectric nanogenerator. J. Phys. Chem. C, 2016, 120(47): 26600-26608.

[56] YAN S, DONG K, LU J, et al. Amphiphobic triboelectric nanogenerators based on silica enhanced thermoplastic polymeric nanofiber membranes. Nanoscale, 2020, 12(7): 4527-4536.

[57] MENDEL N, WU H, MUGELE F. Electrowetting-assisted generation of ultrastable high charge densities in composite silicon oxidefluoropolymer electret samples for electric nanogenerators. Adv. Funct. Mater., 2021, 31: 2007872 